\title{
Aplicación del método Robson en la caracterización de cesáreas realizadas en un hospital de tercer nivel de Pereira
}

\author{
Melissa Piedrahita-Agudelo MD', Juan Esteban Gaviria-Maya, MD, MSc $^{2}$ \\ 1- Médica General Hospital Universitario San Jorge. melipie91710@gmail.com. \\ 2- Especialista en Ginecología y Obstetricia. Docente Universidad Tecnológica de Pereira. Pereira, Colombia.
}

Fecha de recepción: 06/12/2017

Fecha de corrección: 06/08/2018

Fecha de aceptación 04/05/2019

Fecha de publicación 30/06/2019

\begin{abstract}
Resumen
Objetivo: El aumento en cantidad de cesáreas se asocia con morbimortalidad materna. El modelo Robson, utilizado para identificar grupos de mayor aporte a tasas de cesárea, se aplicó en un hospital de tercer nivel para caracterizar grupos de pacientes cesareadas, describir causas obstétricas y desenlaces maternos y fetales.

Materiales y métodos: Estudio de cohorte histórica descriptiva realizado en un hospital de alta complejidad. De 2053 gestantes, se incluyeron 667 cesareadas $(32,4 \%)$ de enero-diciembre de 2014, con datos completos en la historia clínica. Se excluyeron cuando no existió la información para diligenciar el formato de recolección de datos. Se realizó análisis descriptivo utilizando los programas SPSS 20-Excel 2010. Se usó el modelo Robson para calcular tamaño y proporción de cada grupo a la tasa general de cesárea y se describieron causas de cesárea, desenlace materno y neonatal. El estudio lo aprobó el Comité de Ética Hospitalaria.
\end{abstract}

Resultados: Los grupos Robson que más contribuyeron fueron $5 \mathrm{y}$ 10 , con proporciones sobre el total de cesáreas de $25,5 \%$ y $11,2 \%$, respectivamente. Cesárea previa y compromiso del bienestar fetal fueron las principales causas. 99 pacientes presentaron complicaciones relacionadas con cesárea, la infección puerperal fue más frecuente (6.9\%). 173 recién nacidos requirieron unidad neonatal, con dificultad respiratoria como principal complicación (44\%).

Conclusiones: El análisis descriptivo y el modelo Robson permitieron agrupar las pacientes en grupos 2, 5 y 10, además evidenciaron complicaciones maternas $\mathrm{y}$ fetales consistentes con estudios publicados. Esto contribuye a las estadísticas regionales y nacionales.

Palabras clave: Cesárea, clasificación, salas de parto, embarazo.

Copyright (C) Facultad de Ciencias de la Salud de la Universidad Tecnológica de Pereira. 1995-2018. Todos los derechos reservados
Aplication of Robson's method in cesarean sections characterization, in a third level hospital at Pereira

\begin{abstract}
Objective: The increase in number of caesarean sections is associated with maternal morbidity and mortality. The Robson model, used to identify groups with higher contributions at cesarean rates, was applied in a tertiary hospital to characterize groups of patients with highest percentage of cesarean section, describe obstetric causes and maternal and fetal outcomes.
\end{abstract}

Materials and methods: Descriptive historical cohort study conducted in a highly complex hospital. Of 2053 pregnant women, 667 were included $(32.4 \%)$ from January-December 2014, with complete data in the clinical history. They were excluded when there was no information to fill out the data collection format. A descriptive analysis was carried out using the SPSS 20 -Excel 2010 programs. The Robson model was used to calculate the size and proportion of each group at the general cesarean rate and causes of cesarean, maternal and neonatal outcomes were described. The study was approved by the Hospital Ethics Committee.

Results: The Robson groups that contributed the most were 5 and 10 , with proportions over the total cesarean sections of $25.5 \%$ and $11.2 \%$, respectively. Prior cesarean section and commitment to fetal well-being were the main causes. 99 patients presented complications related to cesarean section, puerperal infection was more frequent (6.9\%). 173 newborns required a neonatal unit, with respiratory distress as the main complication (44\%)

Conclusions: The descriptive analysis and the Robson model allowed to group the patients in groups 2,5 and 10 , besides they showed maternal and fetal complications consistent with published studies. This contributes to regional and national statistics.

Keywords: Cesareansection, classification, deliveryrooms, pregnancy

Copyright (C) Facultad de Ciencias de la Salud de la Universidad Tecnológica de Pereira. 1995-2018. Todos los derechos reservados

\section{Introducción}

La cesárea surgió como una alternativa segura en casos de complicaciones asociadas al momento del nacimiento como la desproporción céfalopélvica (DCP), utilización de fórceps o la sinfisiotomía (1), con lo que contribuyó a disminuir la morbimortalidad materno y perinatal (2). 
Desde 1985 la Organización Mundial de la Salud (OMS), estimó que la tasa ideal de cesáreas debería ser menor del $15 \%$. (3) Esto con base en la revisión de los datos procedentes principalmente de países europeos donde, con esta tasa de cesárea, se obtenían resultados maternos y perinatales adecuados. (4) Desde entonces y por diversos motivos, las cesáreas son cada vez más frecuentes, tanto en países desarrollados como en países en desarrollo $(5,6)$. Se evidencia que un aumento mayor al $10 \%$ no se relaciona con una disminución en la morbimortalidad $(7,8)$, y por el contrario el aumento se relaciona con mayores complicaciones maternas y fetales (9-10) además de consecuencias a largo plazo que puede conllevar para la fertilidad y los embarazos posteriores $(4,11)$. Estos riesgos son mayores en las mujeres con escaso acceso a una atención obstétrica integral (5-8-12).

Otro factor importante relacionado con el acceso a la atención materna y neonatal es el costo, puesto que las cesáreas representan un gasto significativo para un sistema de salud sobrecargado y debilitado $(13,15)$.

Con este incremento en la tasas de cesáreas innecesarias y las consecuencias descritas, en los últimos años se ha buscado vencer el desafío de determinar la tasa ideal de cesárea requerida por motivos médicos a la vez que se evitan las intervenciones médicamente innecesarias (4). Es importante resaltar que la proporción de cesáreas a nivel de población es una medida del nivel de acceso a esta intervención y de su uso (4).

En el caso de Colombia en los últimos años se ha notado un incremento en el número de cesaras realizadas, según reportes del DANE ha habido un aumento desde $24,9 \%$ en 1998 hasta $45,7 \%$ en el año 2013 en el número de cesáreas al año (16). Es por eso que la Federación Colombiana de Ginecología (FELCOSOG) junto con la Federación Colombiana de Perinatología (FECOPEN) ha generado estudios con el objetivo de realizar un adecuado acercamiento a esta problemática para generar estrategias encaminadas a la disminución de estas tasas (17).

Una de las principales dificultades que representa definir la tasa ideal es la falta de un sistema intencionalmente aceptado y fiable que genere datos estandarizados que permitan realizar comparaciones e investigar factores que determinen la realización de la cesárea (4). Una revisión sistemática de la literatura encontró 27 clasificaciones para la cesárea y concluyó que el modelo de Robson alcanzó los puntajes más altos de validez desde el punto de vista teórico, y tuvo un alto desempeño en los escenarios clínicos en que se probó (18).

Este sistema propuesto por el Dr. Michael Robson en 2001 (19), estratifica las mujeres según sus características obstétricas y permite comparar las tasas de cesárea en cada grupo con un número menor de factores de confusión (20), además de determinar si las proporciones de cada grupo están de acuerdo o por encima de los patrones internacionales para poder definir áreas de intervención y reducir estas frecuencias altas (21).

A nivel regional no existen estudios en donde se caracterice la población de mujeres gestantes sometidas a cesárea, ni tampoco las razones por las cuales se están realizando las mismas y las complicaciones que se pudieran estar presentando.

El objetivo general del estudio fue caracterizar a los grupos de mujeres gestantes utilizando el método de Robson y determinar las principales causas y desenlaces maternos fetales contribuyendo así a las estadísticas regionales y nacionales con respecto a este tema.

\section{Materiales y métodos:}

Estudio de cohorte histórica descriptiva realizado en el Hospital
Universitario San Jorge (HUSJ), Pereira, Risaralda hospital de alta complejidad que atiende mayoritariamente pacientes del aseguramiento subsidiado por el Estado. Centro de referencia del departamento de Risaralda, Eje Cafetero y norte del Valle del Cauca.

Del total de la población de 2053 gestantes, se incluyeron a todas las mujeres gestantes que fueron sometidas a cesárea en la institución entre enero y diciembre de 2014 y con registro completo en la historia clínica electrónica. A su vez se consideró criterio de exclusión cuando no existió la información completa para diligenciar el formato de recolección de la información establecido por los investigadores, que incluía cuatro grupos grandes de variables sociodemográficas, características del embarazo actual (edad gestacional, posición fetal, categoría única o múltiple, antecedentes ginecoobstétricos, tipo de trabajo de parto), causa obstétrica para definir la cesárea, desenlace materno (días estancia posparto, complicaciones presentadas), desenlace neonatal (APGAR al nacimiento, complicaciones presentadas en el periodo perinatal inmediato y el destino del neonato una vez terminada la cesárea).

Se consideró inicio de trabajo de parto como espontáneo cuando no hubo intervención médica para desencadenar el inicio de las contracciones uterinas, inducido cuando se dio una intervención farmacológica para hacerlo; el antecedente de cicatriz uterina incluyó cesárea o miomectomía previa. La edad gestacional se definió por la ecografía más temprana y en caso de que esta no estuviera disponible por la fecha de última menstruación anotada al momento del ingreso.

La recolección de la información desde las historias clínicas electrónicas se obtuvo a partir de la revisión paciente a paciente de la base de datos aportada por la institución en el periodo del ingreso egreso.

Se obtuvo una muestra final de 667 pacientes que se almacenó en los programas SPSS 20 y Excel 2010 a la cual se le aplicó un análisis descriptivo.

Las pacientes se clasificaron de acuerdo con los diez grupos clínicos, mutuamente excluyentes, previamente descritos por Robson. Se calculó la relación entre las pacientes pertenecientes a cada grupo de Robson con respecto al total de la población atendida ([Número de pacientes atendidas pertenecientes a cada grupo/Número total de pacientes atendidas, tanto vía vaginal como vía cesárea] x 100), lo cual representa el tamaño relativo de cada uno de los grupos. Se cuantificó la proporción de cesáreas realizadas por cada grupo con respecto al total de la población total de cesáreas ([Número de cesáreas realizadas a mujeres pertenecientes a cada grupo/Número de cesáreas] x 100).

El trabajo contó con la autorización del Comité de Ética Hospitalaria del Hospital Universitario San Jorge de Pereira y se garantizó la confidencialidad de la información.

\section{Resultados}

Durante el periodo comprendido entre enero y diciembre de 2014 se atendieron un total de 2053 partos de los cuales, según la base de datos aportada 667 fueron llevados a cesárea (32.4\%). De esta se digitaron el total 667 registros los cuales cumplían los criterios de inclusión fijados previamente. Se dividió la población según características demográficas. Encontrando una media de edad materna de 24.9 años, $81.2 \%$ pacientes con gestación mayor a 37 semanas, $60.1 \%$ multíparas. Según lugar de procedencia, en orden de frecuencia Pereira (335), Dosquebradas (58) y Santa Rosa (48) fueron los principales. 
Tabla 1 parte 1. Distribución de las cesáreas según los grupos de la clasificación de Robson Hospital Universitario San Jorge de Pereira 2014

\section{GRUPOS}

1. Nulíparas, embarazo simple, cefálico, EG mayor o igual a 37 semanas, trabajo de parto espontáneo.

2. Nulíparas, embarazo simple, cefálico, EG mayor o igual 100 a 37 semanas, inducción de trabajo de parto o cesárea antes del trabajo de parto

3. Multíparas sin cicatriz uterina, embarazo simple, cefálico, EG mayor o igual a 37 semanas, trabajo de parto espontáneo.

4. Multíparas sin cicatriz uterina, embarazo simple, cefálico, EG mayor o igual a 37 semanas, inducción de trabajo de parto o cesárea antes del trabajo de parto.
$69 \quad 10,3 \quad 3,36$

$\begin{array}{lll}37 & 5,5 & 1,80\end{array}$
TOTAL CESAREA GRUPO/

11,1

3,60

CESAREA GRUPO/TOTAL

POBLACION (\%)

Tabla 1 parte 2. Distribución de las cesáreas según los grupos de la clasificación de Robson Hospital Universitario San Jorge de Pereira 2014

\begin{tabular}{|c|c|c|c|c|}
\hline & GRUPOS & TOTAL & $\begin{array}{l}\text { CESAREA GRUPO/ } \\
\text { TOTAL CESAREA }(\%)\end{array}$ & $\begin{array}{l}\text { CESAREA GRUPO/TOTAL } \\
\text { POBLACION }(\%)\end{array}$ \\
\hline 1. & $\begin{array}{l}\text { Multíparas con antecedente de al menos una cica- } \\
\text { triz uterina, cefálico, con EG mayor o igual a } 37 \\
\text { semanas }\end{array}$ & 170 & 25,5 & 8,28 \\
\hline 2. & Nulíparas con embarazo simple, podálico. & 42 & 6,3 & 2,05 \\
\hline 3. & $\begin{array}{l}\text { Multíparas con embarazo simple, podálico, con o } \\
\text { sin antecedente de cicatriz uterina. }\end{array}$ & 39 & 5,8 & 1,90 \\
\hline 4. & $\begin{array}{l}\text { Embarazo múltiple, con o sin antecedente de cica- } \\
\text { triz uterina. }\end{array}$ & 41 & 6,1 & 2,00 \\
\hline 5. & $\begin{array}{l}\text { Embarazo simple, en situación transversa u obli- } \\
\text { cua, con o sin antecedente de cicatriz uterina. }\end{array}$ & 20 & 3,0 & 0,97 \\
\hline \multirow[t]{2}{*}{6.} & $\begin{array}{l}\text { Embarazo simple, cefálico, EG de } 36 \text { semanas o } \\
\text { menos, con o sin antecedente de cicatriz uterina. }\end{array}$ & 75 & 11,2 & 3,65 \\
\hline & & 667 & 100,0 & 32 \\
\hline
\end{tabular}

El 40\% (267) de las pacientes eran nulíparas y el 60\% (400) multíparas. 626 (94\%) embarazos fueron únicos y 41 (6\%) gemelares. En 83\% de los trabajos de parto fue de inicio espontáneo y el 17\% restante recibió inducción. El 81\% de los fetos se encontraba en presentación cefálica al momento del parto. El 31\% de las pacientes presentaba cicatriz uterina. La distribución por grupos clínicos de Robson y sus respectivas tasas de cesáreas se resumen en la Tabla 1, donde se identifica que los grupos 5, 2 y 10 son los que presentan porcentajes mayores, por lo cual ameritan una mayor caracterización. 
Tabla 2. Indicación de cesárea y su proporción en el Hospital Universitario San Jorge de Pereira año 2014

COMPLICACIÓN MATERNA RECUENTO (n) PORCENTAJE (\%)

\begin{tabular}{lcc}
\hline Infección puerperal & 46 & 6.9 \\
Admisión a UCI & 23 & 3.4 \\
Hemorragia grave & 14 & 2.4 \\
Dehiscencia o hematoma de la herida quirúrgica & 7 & 1 \\
Histerectomía periparto & 4 & 0.6 \\
Muerte materna & 4 & 0.6 \\
Paro cardiaco & 1 & 0.1 \\
& & 100
\end{tabular}

En la tabla 2 se presentan las indicaciones más comunes de cesárea y su proporción de acuerdo al total de pacientes, según causas maternas y fetales. El antecedente de cesárea previa y trabajo de parto detenido fueron las principales causas maternas, mientras que el compromiso del bienestar y presentación fueron las principales causas fetales.

Tabla 3. Tabla de complicaciones maternas presentadas

\section{INDICACIÓN CESÁREA}

\begin{abstract}
$\longrightarrow$
\end{abstract}
Cesárea previa

Trabajo de parto detenido

Desproporción cefalopélvica

Preclampsia - eclampsia

Enfermedad materna

Abruptio de placenta

Expulsivo prolongado

Pelvis estrecha

Cérvix distócico

Inducción fallida

Ruptura prematura de membranas

Cordón procúbito

Antecedente de óbito

Cuelo desfavorable

\section{CAUSAS MATERNAS}

PORCENTAJE

CAUSAS FETALES

Compromiso del bienestar fetal

Presentación fetal

Macrosomía

Embarazo gemelar

TOTAL

667 
En relación a los dos grupos que presentaron mayor, para las pacientes multíparas con cicatriz uterina previa feto en cefálica y embarazo simple a término (Grupo 5) y pacientes con embarazo simple pretérmino con feto en cefálica (Grupo 10) la cesárea previa y la preclampsia eclampsia fueron las principales causas. (tabla 3 )

Las complicaciones maternas presentadas asociadas a la cesárea para todos los grupos se encuentran en la tabla 6. Otros desenlaces maternos presentados en el posparto fueron la anemia $23(45 \%)$, hipertensión posparto $9(18 \%)$ y síndrome HELLP (del inglés hemolysis, elevated liver enzymes y low platelet count) $5(10 \%) ; 23 \%$ de las pacientes requirieron ingreso a UCI. Se presentaron en total 4 muertes maternas todas asociadas a complicaciones en el posparto por trastornos hipertensivos acompañados de enfermedades autoinmunes como Lupus Eritematoso Sistémico o Síndrome Antifosfolípido.

Con respecto al desenlace neonatal 74\% (492) de los pacientes fueron dados de alta en compañía de sus madres y $26 \%$ (173) requirieron unidad neonatal. Siendo las tres causas principales en orden de frecuencia la dificultad respiratoria en $44 \%$ (76), riesgo séptico en $16 \%$ (28) y la prematurez en $13 \%$ (20). En total se presentaron 4 muertes neonatales, dos de las cuales se asociaron con malformaciones congénitas, una por prematurez y una por asfixia perinatal.

\section{Discusión}

En el estudio realizado se hizo una caracterización de la población de mujeres llevadas a operación cesárea durante el año 2014 Encontrando una media de edad de 24.9 años, procedencia en su mayoría de área urbana, nulíparas, con fetos en posición cefálica, trabajo de parto espontaneo y sin cicatriz uterina previa. Se evidenció una tasa total de cesáreas de $32.4 \%$ sobre el total de mujeres de embarazadas atendidas.

Los grupos de la clasificación de Robson que contribuyeron en mayor número al total de las cesáreas en orden de frecuencia, fueron el grupo 5,2 y el 10 , los cuales representan el $50 \%$ del número total de las pacientes intervenidas, siendo el antecedente de cesárea previa y el compromiso del bienestar fetal las causas maternas y fetales principales. La infección puerperal fue la complicación más reportada como desenlace materno en $6.9 \%$ con 4 muertes maternas reportadas relacionadas con patologías de etiología autoinmune. $26 \%$ de los neonatos requirió unidad neonatal siendo la dificultad respiratoria (44\%) la principal causa.

En relación a las variables sociodemográficas los hallazgos son similares a los encontrados a la descripción demográfica del estudio realizado en Medellín por Zuleta-Tobón (2). Excepto que la población estudiada demostró mayor porcentaje de mujeres multíparas y cicatriz uterina previa, a su vez la posición cefálica se presentó en un menor porcentaje que en el estudio comparado. Tres variables que podrían influir sobre la tasa de cesárea, las dos primeras de manera directa y la tercera de manera indirectamente proporcional.

Con respecto a la tasa de cesárea encontrada en la institución los datos sobrepasan la tasa de cesáreas propuesta hacia 1985 por la Organización Mundial de la Salud (OMS) del 10-15\% (3). En el caso de Colombia para el 2016 se reportó una tasa de cesárea 53.06\%. Mostrando diferencias por departamentos, siendo San Andrés y Sucre los de mayor porcentaje, seguido por toda la costa Atlántica. Para Risaralda se reportó una tasa de cesárea del $28 \%$ (2), cercano a la tasa encontrada en nuestro estudio (32\%), siendo el hospital San Jorge el que mayor población subsidiada atiende en la región.

De acuerdo a datos recientes, la proporción de cesárea es $25 \%$ en Norte América, $30 \%$ en Centro América, más del $30 \%$ en países Europeos y $40 \%$ en los países Latinoamericanos (23), para los países participantes catalogados con un alto índice de desarrollo humano entre los cuales se destacan Brasil, la tasa oscila es de un $44 \%$ (24). Por su parte Países desarrollados como Finlandia, Suecia y Holanda mantienen tasas de cesárea inferiores al $15 \%$ sin compromiso de la tasa de mortalidad perinatal (25).

De acuerdo a los resultados obtenidos, los grupos de la clasificación de Robson que contribuyen en mayor número al total de las cesáreas, son el grupo 5, 2 y 10 , los cuales contribuyen al 50\% del número total de las pacientes intervenidas. Hallazgos consistentes con los presentados para la contribución de estos dos grupos al grupo total de atención de Zuleta-Tobón (2)

El grupo 5 de la clasificación, en nuestro estudio corresponde al mayor número de pacientes. . Esto hace referencia a que las pacientes con antecedente de cesárea previa son llevadas a una cesárea con mayor frecuencia. Un hallazgo adicional y consistente es el alto número de pacientes del grupo 10. En relación a estos resultados un análisis al ajuste de tasas permite ver que el grupo 10, tiene una proporción específica similar a la de otras instituciones (2). Al igual que en el estudio los elementos comunes de los grupos con una proporción elevada de cesárea son la intervención previa, preclampsia, RPM, la calificación del obstetra por cérvix desfavorable o distocia en el parto (2). Con lo cual se sugiere se puede mejorar el entrenamiento y la aplicación de prácticas clínicas basadas en la evidencia en relación a estos temas.

Es de anotar que, aunque no corresponde a un gran porcentaje del total, los grupos $6,7,8$ y 9 , que hace referencia a embarazos con fetos en presentación podálica o situación transversa u oblicua independiente de la paridad, y los embarazos gemelares presentan tasas similares a las reportadas en diversos estudios. En relación a estos grupos es importante tener en cuenta el muy poco impacto en la cifra general, ya que tienen una indicación para el procedimiento, lo que lleva a que de entrada exista un porcentaje de parto por cesárea casi inmodificables (2).

En orden de frecuencia se encontró que la infección puerperal, admisión a UCI y hemorragia grave fueron las tres principales complicaciones maternas presentadas. Datos similares a un estudio de cohorte prospectiva multicéntrica en 2007 en donde se encontró un riesgo significativamente mayor de hemorragia obstétrica $(\mathrm{OR}=$ 1,39; IC $95 \%$ : 1,10-1,76), admisión a unidad de cuidados intensivos $(\mathrm{OR}=2,22$; IC $95 \%: 1,45-3,40)$ y la necesidad de antibióticos posparto $(\mathrm{OR}=5,53$; IC $95 \%: 3,77-8,10)$ en las mujeres sometidas a cesárea comparadas con el parto vaginal (26).

Desde el punto de vista neonatal la complicación más frecuente fue la dificultad respiratoria e ingreso a unidad de cuidado neonatal. Datos consistentes con los reportados en otros estudios en donde reportaron que existe un incremento significativo del riesgo de morbilidad respiratoria neonatal e ingreso a unidad de cuidado intensivo neonatal $(\mathrm{OR}=1,9$; IC 95\% 1,6-2.3) en los recién nacidos por cesárea especialmente en ausencia de trabajo de parto previo (27).

Además de aportar información sobre las características demográficas y descripción de grupos de Robson, este trabajo hace aportes sobre la aplicación del modelo de Robson, el cual se debe tomar como punto de partida para entender más esta problemática. A pesar de que se menciona que no existe un sistema de clasificaron ideal y se propone realizar modelos híbridos que incluyan otras características (28).

Como otros autores que han utilizado el modelo, se propone incluir en futuros trabajos el número de cesáreas previas en las mujeres con este antecedente, así como incluir un juicio con respecto al cumplimiento de los criterios de inducción fallida en las mujeres de los grupos 2 y 4 sometidas a ella (29).

Dentro de las limitaciones del estudio, el carácter retrospectivo de la recolección de la información y la falta de un modelo único de recolección de la información en el momento de registrar las historias 
clínicas en el sistema por parte de los ginecoobstetra son limitantes, no obstante, la disponibilidad de estos registros electrónicos mejora el acceso y la interpretación de los datos.

El presente estudio muestra la utilidad del modelo de Robson como herramienta para realizar una descripción de la población de mujeres llevadas a cirugía cesárea identificando como grupos principales y consistentes con demás estudios los grupos 2- 5 y 10 de Robson los cuales requieren un análisis más detallado para así generar estrategias y de esta manera crear propuestas relacionadas con protocolos de manejo, capacitación de expertos, entre otras que estén orientadas a disminuir la frecuencia del procedimiento. Igualmente se evidencia como de manera consistente las complicaciones maternas y neonatales como infección puerperal, hemorragia y dificultad respiratoria, admisión a unidad de cuidado intensivo neonatal son las más frecuentes asociadas a este procedimiento.

Conflicto de intereses Los autores declaramos que no presentamos conflicto de intereses.

\section{Referencias}

1. Cyr RM. Myth of the ideal cesarean section rate: commentary and historic perspective. Am J Obstet Gynecol. 2006;194:9326.

2. Zuleta-Tobón JJ, Quintero-Rincon F, Quiceo-Ceballos AM. Aplicación del modelo de Robson para caracterizar la realización de cesáreas en una institución de tercer nivel de atención en Medellín, Colombia. Estudio de corte transversal. Rev Colomb Obstet Ginecol. 2013;64:90-99.

3. Appropriate technology for birth. Lancet 1985;2(8452):436-7.

4. Departamento de Salud Reproductiva e Investigación. Organización Mundial de la Salud. Declaración de la OMS sobre tasas de cesárea. 2015. 1:8.

5. Lumbiganon P, Laopaiboon M, Gulmezoglu AM, Souza JP, Taneepanichskul S, Ruyan P, et al. Method of delivery and pregnancy outcomes in Asia: the WHO global survey on maternal and perinatal health 2007- 08. Lancet. 2010;375:4909.

6. Villar J, Carroli G, Zavaleta N, Donner A, Wojdyla D, Faundes A, et al. Maternal and neonatal individual risks and benefits associated with caesarean delivery: multicentre prospective study. BMJ. 2007;335(7628):1025.

7. Villar J, Valladares E, Wojdyla D, Zavaleta N, Carroli G, Velazco A, et al. Caesarean delivery rates and pregnancy outcomes: the 2005 WHO global survey on maternal and perinatal health in Latin America. Lancet. 2006;367:1819-29.

8. Souza JP, Gülmezoglu A, Lumbiganon P, Laopaiboon M, Carroli G, Fawole B, et al. Caesarean section without medical indications is associated with an increased risk of adverse shortterm maternal outcomes: the 2004-2008 WHO Global Survey on Maternal and Perinatal Health. BMC medicine. 2010;8:71

9. Wen SW, Rusen ID, Walker M, Liston R, Kramer MS, Baskett $\mathrm{T}$, et al. Comparison of maternal mortality and morbidity between trial of labor and elective cesarean section among women with previous cesarean delivery. Am J Obstet Gynecol. 2004;191:1263-9.

10. Cárdenas R. [Cesarean-associated complications: the importance of a scarcely justified use]. Gac Med Méx. 2002;138:357-66

11. Federación Colombiana de Obstetricia y Ginecología (Fecolsog), Federación Colombiana de Perinatología (Fecopen). Racionalización del uso de la cesárea en Colombia. Consenso de la Federación Colombiana de Obstetricia y Ginecología (Fecolsog) y la Federación Colombiana de Perinatología (Fecopen) Bogotá, 2014. Rev Colomb Obstet Ginecol. 2014;65:139-151.

12. Villar J, Carroli G, Zavaleta N, Donner A, Wojdyla D, Faundes $\mathrm{A}$, et al. Maternal and neonatal individual risks and benefits associated with caesarean delivery: multicentre prospective study. BMJ. 2007;335(7628):1025
13. Gibbons L, Belizan JM, Lauer JA, Betran AP, Merialdi M, Althabe F. Inequities in the use of cesarean section deliveries in the world. Am J Obstet Gynecol. 2012;206(4):331 e1-19.

14. Connolly ML. High caesarean section figures in Northern Ireland questioned: BBC News Northern Ireland; 2014 [cited 2014]. Available from: http://www.bbc. com/news/uk-northernireland-27195161.

15. What is the right number of caesarean sections? Lancet. 1997;349:815-6.

16. República de Colombia. Departamento Administrativo Nacional de Estadística (DANE). Nacimientos por tipo de parto según departamento de ocurrencia. Disponible en: http//:www.dane. gov.co/

17. Integrantes del Consenso de la Federación Colombiana de Obstetricia y Ginecología (FECOLSOG) y la Federación Colombiana de Perinatología (FECOPEN). Racionalización del uso de cesárea en Colombina, consenso de la Federación Colombiana de Obstetricia y Ginecología (FECOLSOG) y la Federación Colombiana de Perinatología (FECOPEN). Revista Colombiana de Obstetricia y Ginecología. 2014; 65(2): 139151.

18. Torloni MR, Betran AP, Souza JP, Widmer M, Allen T, Gulmezoglu M, et al. Classifications for Cesarean Section: A Systematic Review. PLoS ONE. 2011;6:e14566

19. Robson MS. Classification of caesarean sections. Fetal and Maternal Medicine Review. 2001;12(1):23-39.

20. Monitoring emergency obstetric care: a handbook. Geneva, Switzerland: World Health Organization, 2009.

21. Triunfo S, Ferrazani S, Lanzone A, Scambia, G. Identification of obstetric targets for reducing cesarean section rate using the Robson ten group classification in a tertiary level hospital. Eur J Obstet Gynecol Reprod Biol. 2015;189:91-95.

22. República de Colombia. Departamento Administrativo Nacional de Estadística (DANE). Nacimientos por tipo de parto según departamento de ocurrencia [visitado 2017 noviembre

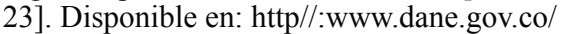

23. Betrán AP, Merialdi M, Lauer JA, Bing-Shun W, Thomas J, Van Look P, et al. Rates of caesarean section: analysis of global, regional and national estimates. Paediatr Perinat Epidemiol 2007;21(2):98-113.

24. Ferreira EC, Pacagnella RC, Costa, ML, Cecatti, JG. The Robson ten-group classification system for appraising deliveries at a tertiary referral hospital in Brazil. Int J Gynaecol Obstet. 2015;129(3):236-239.

25 . Niino $Y$. The increasing cesarean rate globally and what we can do about it. Biosci Trends. 2011;5:139-50.

26. Villar J, Carroli G, Zavaleta N, Donner A, Wojdyla D, Faundes A, et al. World Health Organization 2005. Global Survey on Maternal and Perinatal Health Research Group. Maternal and neonatal individual risks and benefits associated with caesarean delivery: multicentre prospective study. BMJ. 2007;335:1025. Epub 2007 Oct 30.

27. The American College of Obstetricians and Gynecologists (ACOG) - Obstetric Care Consensus. Safe Prevention of Primary Cesarean Delivery. 2014; 123: 693-711.

28. Torloni MR, Betran AP, Souza JP, Widmer M, Allen T, Gulmezoglu M, et al. Classifications for Cesarean Section: A Systematic Review. PLoS ONE. 2011;6:e14566.

29. Zhang J, Troendle J, Reddy UM, Laughon SK, Branch DW, Burkman R, et al. Contemporary cesarean delivery practice in the United States. Am J Obstet Gynecol. 2010;203:326.e1-326. e10. 